\title{
Lenalidomide Causes Selective Degradation of IKZF1 and IKZF3 in Multiple Myeloma Cells
}

\author{
A. Carr ${ }^{2}$, and Benjamin L. Ebert ${ }^{1,2,{ }^{*}}$ \\ ${ }^{1}$ Brigham and Women's Hospital, Boston, USA \\ ${ }^{2}$ Broad Institute of MIT and Harvard, Cambridge, USA \\ ${ }^{3}$ University of Ulm, Germany \\ ${ }^{4}$ Hannover Medical School, Germany \\ ${ }^{5}$ Dana-Faber Cancer Institute, Boston, USA
}

Jan Krönke ${ }^{1,2,3}$, Namrata D. Udeshi ${ }^{2}$, Anupama Narla ${ }^{1}$, Peter Grauman ${ }^{1}$, Slater N. Hurst ${ }^{1}$, Marie McConkey ${ }^{1}$, Tanya Svinkina ${ }^{2}$, Dirk Heckl ${ }^{1,4}$, Eamon Comer $^{2}$, Xiaoyu Li ${ }^{2}$, Christie Ciarlo $^{2}$, Emily Hartman ${ }^{2}$, Nikhil Munshi ${ }^{5}$, Monica Schenone ${ }^{2}$, Stuart L. Schreiber ${ }^{2}$, Steven

\begin{abstract}
Lenalidomide is a drug with clinical efficacy in multiple myeloma and other B cell neoplasms, but its mechanism of action is unknown. Using quantitative proteomics, we found that lenalidomide causes selective ubiquitination and degradation of two lymphoid transcription factors, IKZF1 and IKZF3, by the CRBN-CRL4 ubiquitin ligase. IKZF1 and IKZF3 are essential transcription factors in multiple myeloma. A single amino acid substitution of IKZF3 conferred resistance to lenalidomide-induced degradation and rescued lenalidomide-induced inhibition of cell growth. Similarly, we found that lenalidomide-induced IL2 production in T cells is due to depletion of IKZF1 and IKZF3. These findings reveal a novel mechanism of action for a therapeutic agent, alteration of the activity of an E3 ubiquitin ligase leading to selective degradation of specific targets.
\end{abstract}

Lenalidomide is a highly effective drug for the treatment of multiple myeloma (1) and has activity in other B cell lymphomas. In addition, lenalidomide and its analogues thalidomide and pomalidomide have multiple additional biological effects, including teratogenicity, stimulation of IL-2 production by T cells (2), and inhibition of TNF production by monocytes (3), but the molecular basis of these pleiotropic activities is unknown.

Using an immobilized derivative of lenalidomide in combination with SILAC (Stable Isotope Labeling of Amino Acids in Cell Culture)-based quantitative mass spectrometry (MS), we found that lenalidomide binds DDB1 and CRBN that together with CUL4 and ROC1 form an E3 ubiquitin ligase (CRL4). (Fig. S1) The same target has recently been reported to bind thalidomide, and has been implicated in the teratogenetic effects of

*Corresponding author: Benjamin L. Ebert, bebert@partners.org, Phone: (617) 355-9091, Fax: (617) 355-9193, 1 Blackfan Circle, Karp Building 5.210, Boston, MA 02115. 
thalidomide (4). The finding that CRBN-DDB1 binds both lenalidomide and thalidomide in independent proteomic studies provides powerful evidence that this ubiquitin ligase complex is a major direct protein binding partner for this class of molecules.

We hypothesized that the pleiotropic effects of lenalidomide might be caused by altered ubiquitination of target proteins. Specificity of the CRL4 ubiquitin ligase is mediated by an interchangeable substrate receptor, but no targets have been identified for CRBN, a putative substrate receptor (4-6). To characterize lenalidomide-induced modulation of CRL4-CRBN ubiquitin ligase activity, we used SILAC-based quantitative MS studies to characterize changes in the ubiquitinome and proteome in the MM1S multiple myeloma cell line. Ubiquitination profiling was completed by enrichment of formerly ubiquitinated peptides with an anti-K-ع-GG antibody (Fig. 1A)(7, 8). Two proteins, Ikaros (IKZF1) and Aiolos (IKZF3), scored at the top of the lists of proteins regulated by lenalidomide at both the protein and ubiquitin-site level (Fig. 1B and 1C). Lenalidomide decreased the abundance of IKZF3 $\left(\log _{2}\right.$ ratio -2.09$)$ and IKZF1 $\left(\log _{2}\right.$ ratio -1.54$)$.

While increased ubiquitination would be expected to be associated with decreased protein abundance, we observed a decrease in ubiquitination of multiple lysine residues of IKZF1 and IKZF3 after treating cells with lenalidomide for 12 hours prior to addition of the proteasome inhibitor MG132. A likely interpretation of these results is that IKZF1 and IKZF3 are rapidly ubiquitinated, targeting them for degradation and thereby resulting in a decrease in abundance of both ubiquitinated and absolute levels of these proteins. IKZF1 and IKZF3 also scored at the top of the list of thalidomide-regulated proteins, consistent with the similar biological activity of the molecules (Fig. S2).

In parallel, we examined the landscape of lenalidomide-dependent CRBN protein interactions (Fig. S3). Strikingly, we found that binding of IKZF1 and IKZF3 to the putative CRBN substrate receptor that was enhanced in the presence of lenalidomide (Fig. 1D and S3). As expected, we pulled down all of the members of the CRBN-CRL4 ubiquitin ligase and proteins known to interact with DDB1 including subunits 1 to 8 of the COP9 signalosome complex in both, untreated or lenalidomide treated cells. No other known substrate receptors for DDB1 were co-immunoprecipitated, indicating that CRBN is a substrate receptor and precludes binding of alternative receptors to DDB1. In aggregate, the proteomic data suggest that lenalidomide increases the binding of IKZF1 and IKZF3 to the CRBN-DDB1 ubiquitin ligase complex, leading to increased ubiquitination and consequent degradation.

To validate this putative mechanism, we analyzed whether lenalidomide causes posttranscriptional regulation of IKZF1 and IKZF3 protein abundance. The cDNAs of candidate genes, fused to firefly luciferase (FFluc), were expressed in 293T cells (9). IKZF1 and IKZF3 conferred a lenalidomide-regulated decrease in luciferase activity. In contrast, luciferase levels were not altered after lenalidomide treatment when FFluc was fused to RAB28, a protein that decreased in abundance after lenalidomide treatment but did not bind to CRBN. Similarly, lenalidomide did not alter the abundance of FFluc fused to three other transcription factors of the Ikaros family, Helios (IKZF2), Eos (IKZF4) and Pegasus (IKZF5); IRF4, a protein implicated in lenalidomide activity (10); or the transcription 
factors HOXA9 and Myc (Fig. 2A). We confirmed that, in MM1S cells stably expressing HA-IKZF1 or HA-IKZF3, lenalidomide caused a dose-dependent reduction of both proteins (Fig. S5). Taken together, these results demonstrate the selective regulation of IKZF1 and IKZF3 levels in response to lenalidomide.

We next examined endogenous protein expression in response to lenalidomide. Lenalidomide strongly decreased the abundance of IKZF1 and IKZF3 in a dose-dependent manner in cell lines (Fig. 2B+S4A) as well as in primary multiple myeloma samples (Fig. $2 \mathrm{E}$ ), as did thalidomide and pomalidomide (Figure S4C). Depletion of these proteins was evident in as little as 3 hours after lenalidomide treatment (Fig. 2C). In contrast, IKZFI and $I K Z F 3$ mRNA levels were not altered by lenalidomide treatment (Figure 2D+S4B).

Lenalidomide induced ubiquitination of HA-tagged IKZF1 and IKZF3 expressed in MM1S (Fig. 2F) and 293T cells as well as endogenous IKZF1 and IKZF3 (Fig. S6). Cullin-RING ubiquitin ligase (CRL) activity depends on NEDDylation, (11) and treatment with the Nedd8 enzyme inhibitor MLN-4924 prevented the lenalidomide-induced decrease of IKZF1 and IKZF3 (Fig. S5). These experiments demonstrate that lenalidomide-induced degradation of IKZF1 and IKZF3 involves ubiquitination by a cullin-based E3 ubiquitin ligase.

We next sought to determine whether lenalidomide-induced ubiquitination of IKZF1 and IKZF3 is caused by altered binding of these proteins to CRBN, as observed in our proteomic studies. We confirmed that more IKZF1 and IKZF3 co-immunoprecipitated with endogenous CRBN in cells treated with lenalidomide (Fig. 3A). If CRBN is essential for lenalidomide-induced degradation of IKZF1 and IKZF3, then loss or mutation of CRBN would inhibit the effect of the drug. Knockdown of CRBN by shRNAs as well as expression of the CRBN ${ }^{\text {YWAA }}$ mutant (4) that does not bind lenalidomide abrogated degradation of IKZF1 and IKZF3 and conferred lenalidomide resistance to MM1S cells (Fig. S7), consistent with previous studies that have shown CRBN to be essential for lenalidomide activity in multiple myeloma $(12,13)$. IKZF3 was ubiquitinated in vitro when coimmunoprecipitated with CRBN from 293T cells, demonstrating that IKZF3 is an enzymatic substrate of the CRBN complex(Fig. 3B). These studies demonstrate that lenalidomide causes increased binding of IKZF1 and IKZF3 to CRBN and promotes their ubiquitination and degradation.

In order to identify a degron sequence in IKZF3 responsible for lenalidomide sensitivity, we generated a series of deletion mutants and identified a 58 amino acid sequence that is sufficient for lenalidomide sensitivity, of which 30 amino acids are essential (Fig. 3CD+S8). The critical amino acid sequence lies within zinc finger domain 2, which is highly homologous between Ikaros proteins. IKZF2 and IKZF4, which are not sensitive to lenalidomide, differ from IKZF1 and IKZF3 at only one position within this critical region (Fig. 3D). Substitution of Q147 in IKZF3 with a histidine residue $\left(I K Z F 3^{\mathrm{Q} 147 \mathrm{H}}\right)$, which is present at this corresponding site in IKZF2 and IKZF4, caused resistance to lenalidomideinduced degradation. Conversely, when the corresponding histidine (H188) in IKZF4 was changed to glutamine $\left(I K Z F 4^{\mathrm{H} 188 \mathrm{Q}}\right)$, IKZF4 was degraded after lenalidomide treatment (Fig. 3D). Binding to CRBN in the presence of lenalidomide is decreased for IKZF3 Q147H compared to wildtype IKZF3 (Figure S8C). This domain is therefore necessary and 
sufficient for lenalidomide-induced binding to CRBN and subsequent protein degradation, and amino acid changes in this region provide the basis for differential sensitivity to lenalidomide between Ikaros family members.

Having demonstrated that lenalidomide regulates IKZF1 and IKZF3 ubiquitination and abundance, we next sought to determine whether these proteins mediate specific biological and therapeutic effects of lenalidomide. IKZF1 and IKZF3 are essential transcription factors for terminal differentiation of B and T cell lineages $(14,15)$. While IKZF1 is highly expressed in early lymphoid progenitors, IKZF3 is expressed at high levels in more mature B cell neoplasms $(16,17)$, and murine studies have demonstrated that IKZF3 is required for the generation of plasma cells, the physiologic counterparts of multiple myeloma cells (18). Therefore, we examined the dependence of multiple myeloma cells on IKZFI and IKZF3 expression by genetic silencing of these proteins using RNA interference. IKZFI and IKZF3 shRNAs that effectively decrease expression of the target proteins (Fig. S11) inhibited growth of lenalidomide-sensitive multiple myeloma cell lines, while lenalidomide insensitive cell lines were unaffected (Fig. 4A+S9). Similarly, expression of a dominant negative IKZF3 isoform that lacks the complete DNA binding region resulted in depletion of MM1S cells (Fig. 4B). Over-expression of IKZF3 conferred relative lenalidomide-resistance to MM1S cells when competed with MM1S cells infected with a control retrovirus (Fig. S9B). Moreover, MM1S cells expressing the lenalidomide-resistant IKZF3Q147H mutation were relatively resistant towards lenalidomide when competed against MM1S cells expressing wild-type IKZF3 (Fig. 4B). These studies indicate that the anti-proliferative effect of lenalidomide in multiple myeloma cells is mediated by depletion of IKZF1 and IKZF3.

The transcription factor IRF4 was previously reported to be an essential gene in multiple myeloma, and was implicated in the activity of lenalidomide in this disease $(10,19)$. While IRF4 protein levels were only slightly decreased in our protoemic analysis after 12 hours treatment, we observed a significant decrease of IRF4 mRNA and protein when cells were treated for 24 hours and longer. Knockdown of IKZF3 also suppressed IRF4 mRNA levels, suggesting that IRF4 is a transcriptional target of IKZF3 (Fig. S10).

IKZF3 binds the IL2 gene promoter and represses IL2 transcription in T cells (20,21). We therefore sought to determine whether lenalidomide regulates IL2 levels by modulating IKZF3 expression. Both IKZF1 and IKZF3 protein levels decrease markedly in primary human T cells treated with lenalidomide (Fig. 4D). Lenalidomide induced IL2 mRNA expression by 3.3-fold in T cells expressing a control shRNA. IL2 expression was induced by shRNA-mediated $I K Z F 3$ or IKZFI knockdown and could not be further enhanced by lenalidomide (Fig. 4E). Similarly, the effect of lenalidomide on IL2 expression was abrogated by shRNA knockdown of $C R B N$ (Fig. 4F). These studies demonstrate that induction of IL2 is mediated by de-repression of the IL2 promoter by depletion of IKZF3.

In aggregate, our studies demonstrate that lenalidomide acts via a novel mechanism of drug activity, enforced binding of the substrate receptor CRBN to IKZF1 and IKZF3, resulting in selective ubiquitination and degradation of the target proteins. IKZF1 and IKZF3 play central roles in the biology of $\mathrm{B}$ and $\mathrm{T}$ cells, and ablation of protein expression for these 
transcription factors explains the activity of lenalidomide in lymphoid cells. In particular, IKZF3 is critical for plasma cell development, and our data indicate that IKZF3 is essential in multiple myeloma, a plasma cell malignancy, providing a mechanistic basis for therapeutic efficacy in this disorder (18). Moreover, the activity of lenalidomide in other B cell neoplasms, including mantle cell lymphoma (22) and chronic lymphocytic leukemia (23), may be explained by high IKZF3 expression in these disorders (16). Somatic inactivation of $I K Z F 1$ and $I K Z F 3$ occurs in acute lymphoblastic leukemia, resulting in an accumulation of immature lymphoid progenitor cells, consistent with an essential role for these factors in lympohid differentiation $(24,25)$. In T cells, ablation of IKZF3-mediated repression of IL2 gene expression provides a mechanism for increased IL2 production in response to lenalidomide. The teratogenicity of thalidomide and the efficacy of lenalidomide in myelodysplastic syndrome may be mediated by alternative substrates in different cellular lineages.

RING-based E3 ubiquitin ligases are characterized by a high specificity for their substrates and therefore represent promising drug targets (26). Our studies reveal that lenalidomide modulates the activity of the CRL4-CRBN complex to increase ubiquitination of two transcription factors, IKZF1 and IKZF3, which would otherwise be considered "undruggable." A plant hormone, auxin, appears to act similarly, increasing the interaction between a ubiquitin ligase and a specific substrate, suggesting that this mechanism might be operative in additional biological contexts (27). Selective ubiquitination and degradation of specific targets provides a novel mechanism of therapeutic activity for proteins that are not otherwise amenable to small-molecule inhibition.

\section{Supplementary Material}

Refer to Web version on PubMed Central for supplementary material.

\section{Acknowledgments}

We thank Yu-Tzu Tai (Dana-Faber Cancer Institute) for technical assistance with primary multiple myeloma samples. This work was supported by funding from the NIH (R01HL082945, P01 CA108631), a Leukemia and Lymphoma Society Scholar Award, an Evans Foundation grant and a grant from the Starr Cancer Consortium to BLE. In addition, the work was supported by a grant from the SPARC consortium and by an NHGRI (RL1HG004671) initiative in genome-based drug discovery. JK was supported by the German Research Foundation (DFG, Kr-3886/1-1). DH was supported by the German Cancer Aid.

\section{References}

1. Rajkumar SV, et al. Combination therapy with lenalidomide plus dexamethasone (Rev/Dex) for newly diagnosed myeloma. Blood. Dec 15.2005 106:4050. [PubMed: 16118317]

2. Haslett PA, Corral LG, Albert M, Kaplan G. Thalidomide costimulates primary human T lymphocytes, preferentially inducing proliferation, cytokine production, and cytotoxic responses in the CD8+ subset. J Exp Med. Jun 1.1998 187:1885. [PubMed: 9607928]

3. Sampaio EP, Sarno EN, Galilly R, Cohn ZA, Kaplan G. Thalidomide selectively inhibits tumor necrosis factor alpha production by stimulated human monocytes. J Exp Med. Mar 1.1991 173:699. [PubMed: 1997652]

4. Ito T, et al. Identification of a primary target of thalidomide teratogenicity. Science. Mar 12.2010 327:1345. [PubMed: 20223979] 
5. Groisman R, et al. The ubiquitin ligase activity in the DDB2 and CSA complexes is differentially regulated by the COP9 signalosome in response to DNA damage. Cell. May 2.2003 113:357. [PubMed: 12732143]

6. Angers S, et al. Molecular architecture and assembly of the DDB1-CUL4A ubiquitin ligase machinery. Nature. Oct 5.2006 443:590. [PubMed: 16964240]

7. Udeshi ND, Mertins P, Svinkina T, Carr SA. Large-scale identification of ubiquitination sites by mass spectrometry. Nature protocols. Oct.2013 8:1950.

8. Udeshi ND, et al. Refined preparation and use of anti-diglycine remnant (K-epsilon-GG) antibody enables routine quantification of 10,000s of ubiquitination sites in single proteomics experiments. Mol Cell Proteomics. Mar.2013 12:825. [PubMed: 23266961]

9. Koivunen P, et al. Transformation by the (R)-enantiomer of 2-hydroxyglutarate linked to EGLN activation. Nature. Mar 22.2012 483:484. [PubMed: 22343896]

10. Yang Y, et al. Exploiting synthetic lethality for the therapy of ABC diffuse large B cell lymphoma. Cancer Cell. Jun 12.2012 21:723. [PubMed: 22698399]

11. Soucy TA, et al. An inhibitor of NEDD8-activating enzyme as a new approach to treat cancer. Nature. Apr 9.2009 458:732. [PubMed: 19360080]

12. Lopez-Girona A, et al. Cereblon is a direct protein target for immunomodulatory and antiproliferative activities of lenalidomide and pomalidomide. Leukemia. May 3.2012

13. Zhu YX, et al. Cereblon expression is required for the antimyeloma activity of lenalidomide and pomalidomide. Blood. Nov 3.2011 118:4771. [PubMed: 21860026]

14. Georgopoulos K, et al. The Ikaros gene is required for the development of all lymphoid lineages. Cell. Oct 7.1994 79:143. [PubMed: 7923373]

15. Cortes M, Wong E, Koipally J, Georgopoulos K. Control of lymphocyte development by the Ikaros gene family. Current opinion in immunology. Apr.1999 11:167. [PubMed: 10322160]

16. Nuckel H, et al. The IKZF3 (Aiolos) transcription factor is highly upregulated and inversely correlated with clinical progression in chronic lymphocytic leukaemia. Br J Haematol. Jan.2009 144:268. [PubMed: 19016725]

17. Morgan B, et al. Aiolos, a lymphoid restricted transcription factor that interacts with Ikaros to regulate lymphocyte differentiation. The EMBO journal. Apr 15.1997 16:2004. [PubMed: 9155026]

18. Cortes M, Georgopoulos K. Aiolos is required for the generation of high affinity bone marrow plasma cells responsible for long-term immunity. J Exp Med. Jan 19.2004 199:209. [PubMed: 14718515]

19. Shaffer AL, et al. IRF4 addiction in multiple myeloma. Nature. Jul 10.2008 454:226. [PubMed: 18568025]

20. Gandhi R, et al. Activation of the aryl hydrocarbon receptor induces human type 1 regulatory $\mathrm{T}$ cell-like and Foxp3(+) regulatory T cells. Nature immunology. Sep.2010 11:846. [PubMed: 20676092]

21. Quintana FJ, et al. Aiolos promotes TH17 differentiation by directly silencing Il2 expression. Nature immunology. Aug.2012 13:770. [PubMed: 22751139]

22. Habermann TM, et al. Lenalidomide oral monotherapy produces a high response rate in patients with relapsed or refractory mantle cell lymphoma. Br J Haematol. May.2009 145:344. [PubMed: 19245430]

23. Chanan-Khan A, et al. Clinical efficacy of lenalidomide in patients with relapsed or refractory chronic lymphocytic leukemia: results of a phase II study. J Clin Oncol. Dec 1.2006 24:5343. [PubMed: 17088571]

24. Mullighan CG, et al. Genome-wide analysis of genetic alterations in acute lymphoblastic leukaemia. Nature. Apr 12.2007 446:758. [PubMed: 17344859]

25. Winandy S, Wu P, Georgopoulos K. A dominant mutation in the Ikaros gene leads to rapid development of leukemia and lymphoma. Cell. Oct 20.1995 83:289. [PubMed: 7585946]

26. Deshaies RJ, Joazeiro CA. RING domain E3 ubiquitin ligases. Annual review of biochemistry. 2009; 78:399. 
27. Tan X, et al. Mechanism of auxin perception by the TIR1 ubiquitin ligase. Nature. Apr 5.2007 446:640. [PubMed: 17410169]

\section{Supplement References}

28. Ong SE, Mann M. A practical recipe for stable isotope labeling by amino acids in cell culture (SILAC). Nature protocols. 2006; 1:2650.

29. Bendall SC, et al. Prevention of amino acid conversion in SILAC experiments with embryonic stem cells. Mol Cell Proteomics. Sep.2008 7:1587. [PubMed: 18487603]

30. Rappsilber J, Mann M, Ishihama Y. Protocol for micro-purification, enrichment, pre-fractionation and storage of peptides for proteomics using StageTips. Nature protocols. 2007; 2:1896.

31. Koivunen $\mathrm{P}$, et al. Transformation by the (R)-enantiomer of 2-hydroxyglutarate linked to EGLN activation. Nature. Mar 22.2012 483:484. [PubMed: 22343896]

32. Ong SE, et al. Identifying the proteins to which small-molecule probes and drugs bind in cells. Proc Natl Acad Sci U S A. Mar 24.2009 106:4617. [PubMed: 19255428]

33. Angers S, et al. Molecular architecture and assembly of the DDB1-CUL4A ubiquitin ligase machinery. Nature. Oct 5.2006 443:590. [PubMed: 16964240] 
A

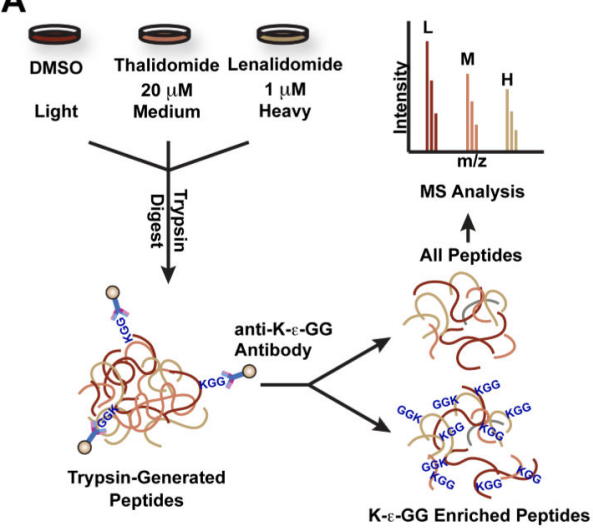

C

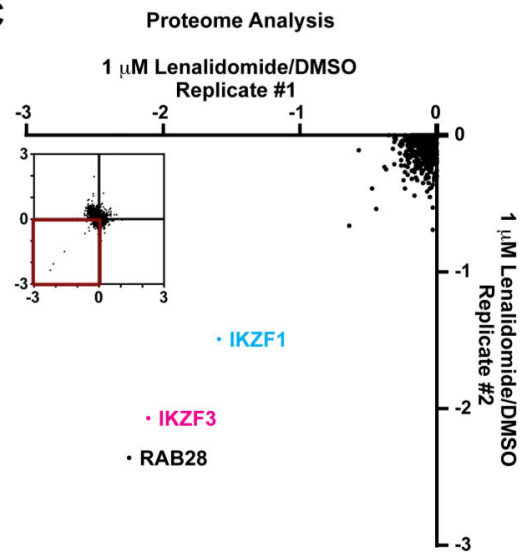

B

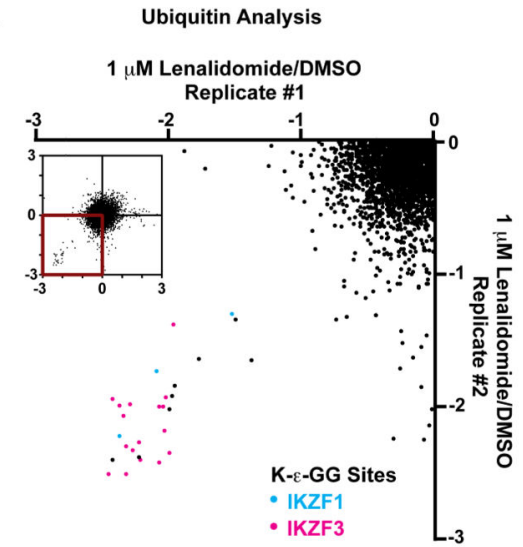

D

CRBN Interaction

in the Presence of Lenalidomide

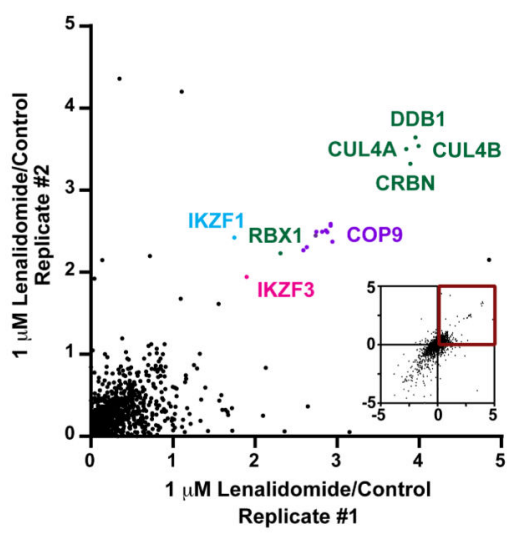

Fig. 1.

Proteomic analysis of lenalidomide-induced changes in ubiquitination, protein abundance and CRBN interaction in MM1S cells. (A) Experimental design for SILAC-based assessment of global changes in ubiquitination and protein levels. Cells were treated for 12 hours with DMSO, lenalidomide, or thalidomide. For ubiquitination analysis $5 \mu \mathrm{M}$ MG132 were added for the last 3 hours. (B) $\log _{2}$ ratios for individual K- $\varepsilon$-GG sites of lenalidomide versus DMSO treated cells for replicate 1 and 2. Each dot represents a unique K- $\varepsilon$-GG site. (C) $\log _{2}$ ratios of changes of protein abundance of lenalidomide versus DMSO treated cells. Each dot represents a distinct protein group. (D) CRBN interaction analysis in cells treated for 6 hours with $1 \mu \mathrm{M}$ lenalidomide. Scatter plot shows $\log _{2}$ changes of proteins pulled down by HA-CBRN in lenalidomide versus DMSO treated control cells. 
A
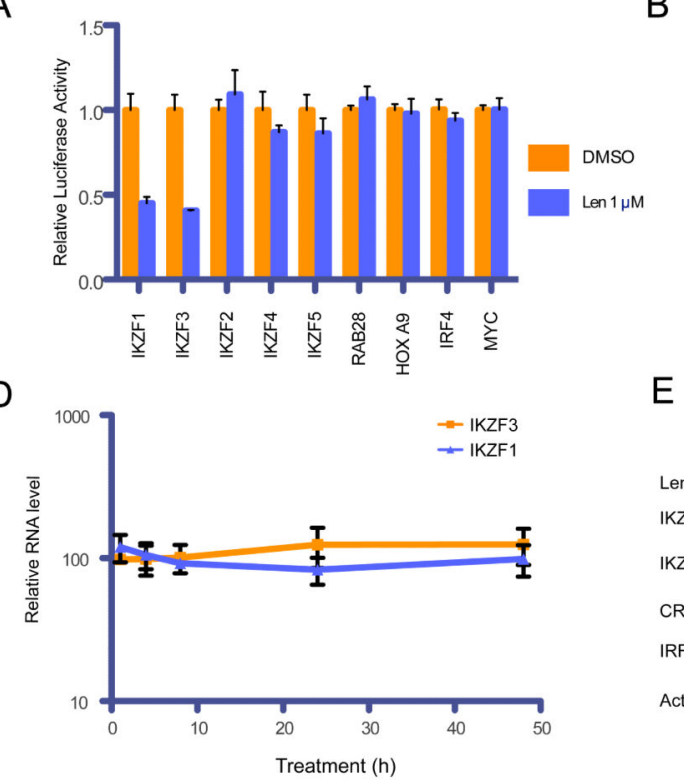

B

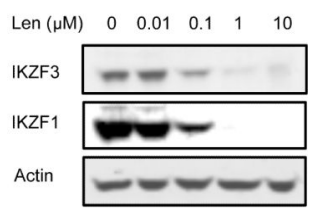

E

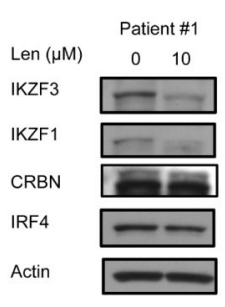

C

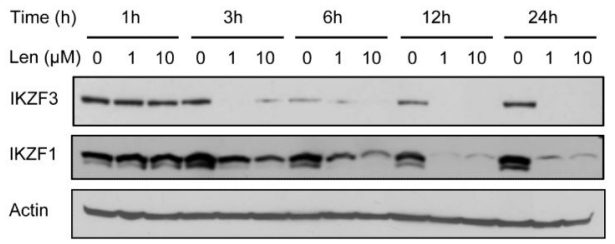

$\mathrm{F}$

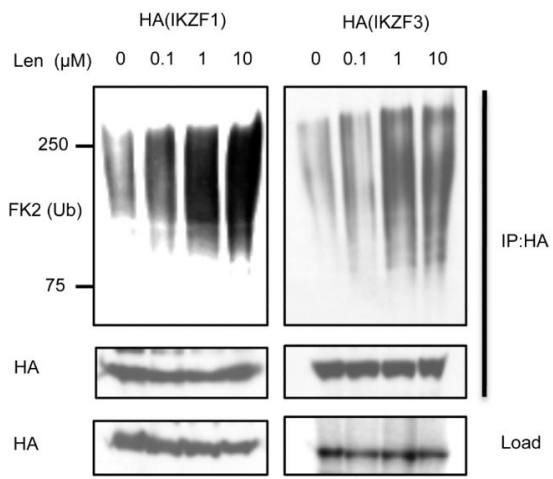

Fig. 2.

Effect of lenalidomide on IKZF1 and IKZF3 protein levels. (A) 293T cells transfected with vectors expressing the indicated cDNA fused to firefly luciferase and control renilla luciferase were treated with DMSO or $1 \mu \mathrm{M}$ lenalidomide for 24 hours. Bars represent the firefly to renilla luciferase ratio, normalized to DMSO-treated cells. (B) Effects of lenalidomide on endogenous IKZF1 and IKZF3 in MM1S cells treated for 24 hours. (C) Time course of lenalidomide treatment in MM1S cells for IKZF1 and IKZF3 protein levels and (D) mRNA levels. (E) Primary multiple myeloma samples were treated for 6 hours and analyzed by immunoblot. (H) In vivo ubiquitination analysis of HA-tagged IKZF1 and IKZF3 expressed in MM1S cells treated for 1.5 hours with $100 \mathrm{nM}$ Epoxomicin and the indicated concentrations of lenalidomide. The FK2 antibody detects covalently linked ubiquitin. 


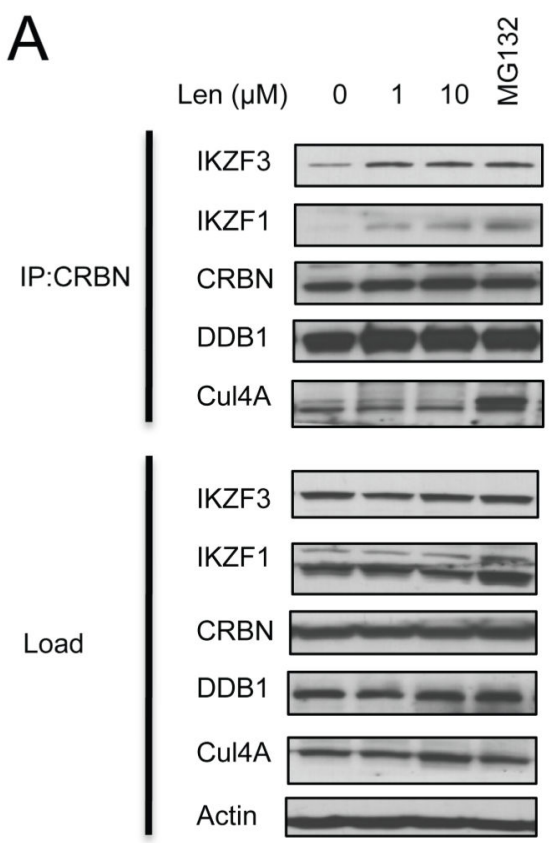

C
B

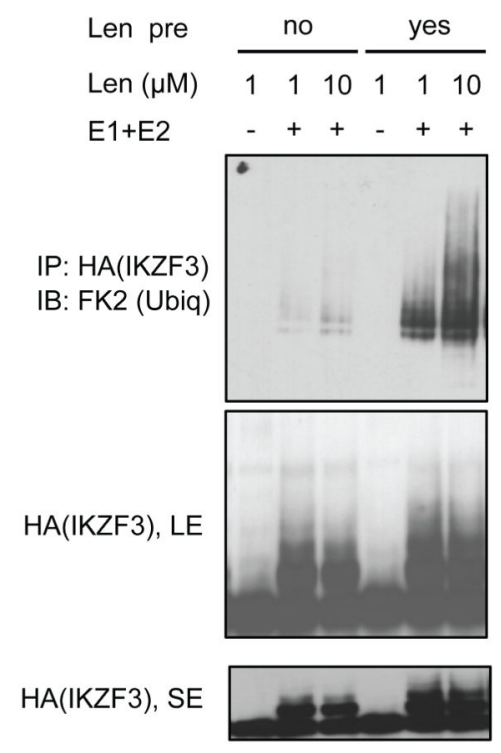

$\mathrm{D}$

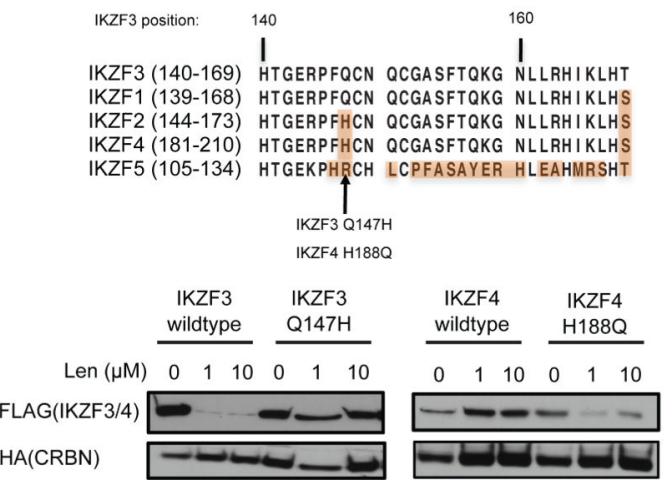

Fig. 3.

CRBN is a substrate receptor for IKZF1 and IKZF3. (A) Immunoprecipitiation of endogenous CRBN in MM1S cells treated for 1 hour with the indicated drugs. (B) In vitro ubiquitination reaction of HA-IKZF3 co-immunoprecipitated by FLAG-CRBN from 293T cells and incubated in the presence or absence of $\mathrm{E} 1$ and $\mathrm{E} 2$ ubiquitin conjugating enzymes. (C) Mapping of the degron that confers lenalidomide sensitivity. Blue boxes in the IKZF3 protein represent zinc finger domains. (D) Sequence alignment of the core lenalidomide degron between the 5 Ikaros proteins. Western blots of 293T cells lysates 48 hours after cotransfection of FLAG-tagged IKZF3 or IKZF4 with HA-tagged CRBN and 24 hours drug treatment. 
A

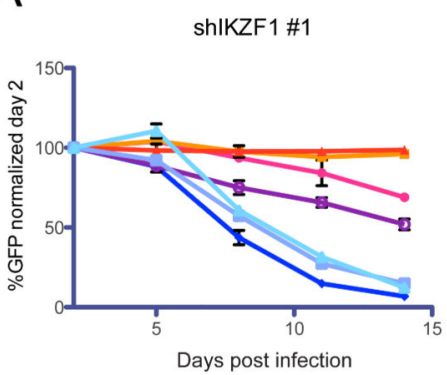

B

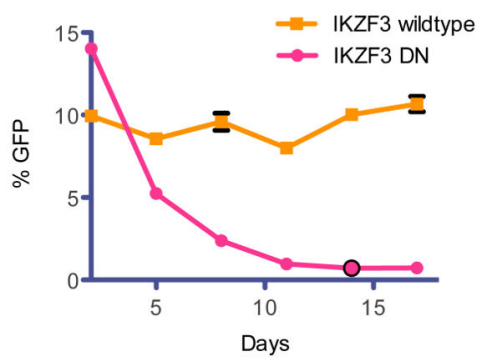

shIKZF3 \#1

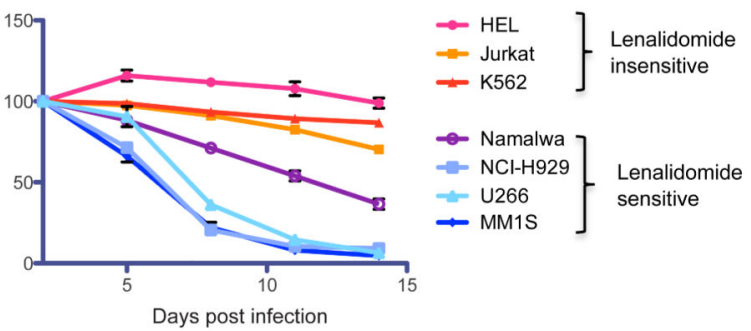

C

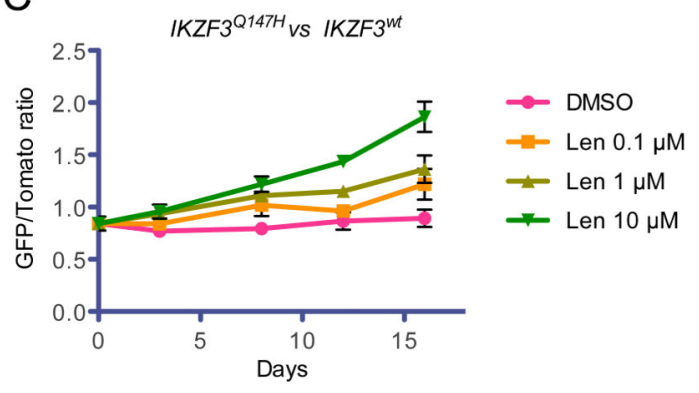

D

$\mathrm{E}$

CD3+ T cells
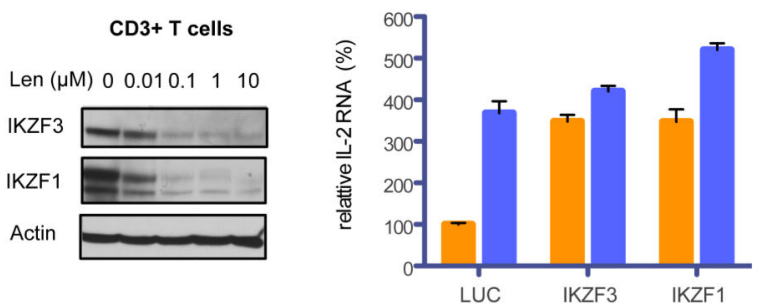

$\mathrm{F}$

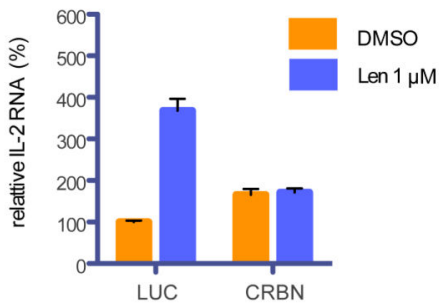

Fig. 4.

Biological role of IKZF1 and IKZF3 in multiple myeloma cell lines and T cells. (A) Lenalidomide-sensitive and insensitive cell lines were infected with lentivirus expressing $I K Z F 1$ or $I K Z F 3$ shRNAs and GFP. Relative depletion was assessed by flow cytometry and normalized to day 2 post-infection. (B) MM1S cells were transduced with retrovirus expressing GFP and wild-type $I K Z F 3$ or a dominant negative $I K Z F 3$ isoform with deletion of the complete DNA binding region. (C) MM1S cells were infected with retrovirus expressing IKZF3 $3147 \mathrm{H} / \mathrm{GFP}$ or IKZF3wt/dTomato and competed against each other in media containing DMSO or lenalidomide. (D) Human CD3+ T cells were stimulated with plate-bound anti-CD3 and anti-CD28 and treated with different concentrations of lenalidomide for 24 hours. (E+F) T cells were infected with lentiviral vectors expressing shRNAs targeting the indicated genes. After selection with puromycin, T cells were stimulated with anti-CD3/CD28 Dynabeads and treated with DMSO or $1 \mu \mathrm{M}$ lenalidomide for 12 hours before lysis. IL-2 RNA expression levels were analyzed by quantitative RTPCR using GAPDH expression as an internal control. 